\title{
Methylation of the calcium channel regulatory subunit $\alpha 2 \delta$-3 (CACNA2D3) predicts site-specific relapse in oestrogen receptor-positive primary breast carcinomas
}

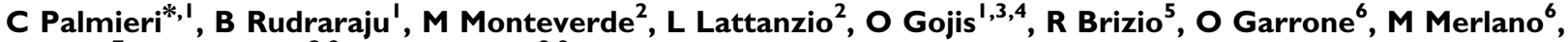 N Syed ${ }^{7}$, C Lo Nigro ${ }^{2,9}$ and T Crook ${ }^{8,9}$}

'Cancer Research UK Laboratories, Imperial Centre for Translational and Experimental Medicine, Division of Cancer, Imperial College London, Du Cane Road, London WI 2 ONN, UK; ${ }^{2}$ Laboratory of Cancer Genetics and Translational Oncology, S. Croce General Hospital, Cuneo, Italy; ${ }^{3}$ Department of Gynaecology and Obstetrics, Third Faculty of Medicine, Charles University in Prague, Ruska 87, Prague 10100 00, Czech Republic; ${ }^{4}$ Department of Pathology, Third Faculty of Medicine, Charles University in Prague, Ruska 87, Prague 10100 00, Czech Republic; ${ }^{5}$ Department of Pathology, S. Croce General Hospital, Cuneo, Italy; ${ }^{6}$ Medical Oncology, Oncology Department, S. Croce General Hospital, Cuneo, Italy; ${ }^{7}$ Neuro-oncology Laboratory, Imperial College Healthcare NHS Trust, Charing Cross Hospital, London W6 8RF, UK; ${ }^{8}$ Dundee Cancer Centre, Ninewells Hospital, Dundee, UK

\begin{abstract}
BACKGROUND: Calcium is an important intracellular messenger that mediates many biological processes that are relevant to the malignant process. Calcium ion channels are key in controlling the intracellular calcium, and little is known about their role in human cancer.

METHODS: We used qPCR and pyrosequencing to investigate expression and epigenetic regulation of the calcium channel regulatory subunit $\alpha_{2} \delta-3$ (CACNA2D3) in breast cancer cell lines, primary cancers and metastatic lesions.

RESULTS: Expression of CACNA2D3 mRNA is regulated in breast cancer cell lines by methylation in the CpG island located in the $5^{\prime}$ regulatory region of the gene. Expression is upregulated by azacytidine (AZA) in cells with CpG island methylation but unaffected in cells lacking methylation. In primary breast carcinomas, methylation is more common in cancers, which subsequently relapse with loco-regional and, particularly, visceral metastatic disease in both oestrogen receptor- $\alpha$ (ER)-positive and -negative cases. Furthermore, CACNA2D3 CpG island is frequently methylated in breast cancer that has metastasised to the central nervous system. CONCLUSION: Methylation-dependent transcriptional silencing of CACNA2D3 may contribute to the metastatic phenotype of breast cancer. Analysis of methylation in the CACNA2D3 CpG island may have potential as a biomarker for risk of development of metastatic disease.
\end{abstract}

British Journal of Cancer (2012) 107, 375-381. doi:I0.1038/bjc.2012.231 www.bjcancer.com

Published online 29 May 2012

(C) 2012 Cancer Research UK

Keywords: breast cancer; metastasis; calcium channels; epigenetics

The calcium ion $\left(\mathrm{Ca}^{2+}\right)$ is a key intracellular messenger and regulates a diverse range of cellular processes by activating or inhibiting cellular signalling pathways and $\mathrm{Ca}^{2+}$-regulated proteins. These processes range from muscle contraction to apoptosis (Berridge et al, 2003; Monteith et al, 2007). Calcium ion has been implicated either directly or indirectly in many of the essential alterations key for malignant growth (Hanahan and Weinberg, 2000), having been shown to be involved in proliferation (Becchetti, 2011), cell motility, (Huang et al, 2004), angiogenesis (Patton et al, 2003), resistance to apoptosis (Rizzuto et al, 2003) and transcriptional regulation (Rizzuto and Pozzan, 2006). These effects could be modulated by changes in plasma membrane $\mathrm{Ca}^{2+}$ channel expression, $\mathrm{Ca}^{2+}$ efflux pumps as well as the expression of proteins that control the $\mathrm{Ca}^{2+}$ content of the endoplasmic reticulum (Monteith et al, 2007). Epigenetic mechanisms have been reported as one of the potential causes for changes in the

\footnotetext{
*Correspondence: Dr C Palmieri; E-mail: c.palmieri@imperial.ac.uk

${ }^{9}$ These authors contributed equally to this work.

Received 7 March 2012; revised 23 April 2012; accepted 26 April 2012; published online 29 May 2012
}

expression of specific $\mathrm{Ca}^{2+}$ pumps and channels in human cancers (Toyota et al, 1999; Kim et al, 2003; Paz et al, 2003).

Voltage-gated $\left(\mathrm{Ca}_{\mathrm{V}}\right)$ calcium channels of the $\mathrm{Ca}_{\mathrm{V}} 1$ and $\mathrm{Ca}_{\mathrm{V}} 2$ classes exist as heteromeric complexes, that is composed of a poreforming $\alpha 1$ subunit and three regulatory subunits, $\alpha 2 \delta, \beta$ and $\delta$ (Davies et al, 2007). There are four calcium channel voltagedependent $\alpha 2 \delta$ subunit genes, CACNA2D1 to CACNA2D4 (Qin et al, 2002). The importance of calcium ion channels in the malignant process has previously been shown by CACNA2D2 subunit, which is located in 3 p21.3 region, a region deleted in breast and lung cancer (Wei et al, 1996). It has been shown to be expressed in normal lung tissue but lost in malignant tissue and its presence has been shown to mediate apoptosis (Carboni et al, 2003).

The CACNA2D3 subunit has been implicated to have a role in a number of cancers. An 80 -fold decrease in expression of CACNA2D3 has been reported in the highly metastatic osteosarcoma cell line MG63-A1, when compared with the parental cell line (Xiong et al, 2009). CACNA2D3 is a putative tumour suppressor gene in both lung cancer, renal cell cancer neuroblastoma and squamous cell oesophageal cancer (Hanke et al, 2001; 
De Preter et al, 2006; Tai et al, 2006; Qin et al, 2008). CACNA2D3 is highly expressed in neuroblasts and favourable prognosis neuroblastomas, with expression downregulated in unfavourable neuroblastomas (De Preter et al, 2006; Thorell et al, 2009). Comparison of 14 pairs of primary gastric carcinomas with matched normal gastric mucosa found that the CACNA2D3 $\mathrm{CpG}$ island was unmethylated in all 14 normal tissues, whereas methylation was present in $36 \%$ of primary tumours. Assessment of a large group of unrelated gastric cancer and normal tissue found that the CACNA2D3 CpG island was methylated in $30 \%$ of primary tumours, and $5.3 \%$ of normal tissue (Wanajo et al, 2008). A similar rate of methylation was reported in an independent cohort of gastric cancers (Yuasa et al, 2009). A significantly reduced survival time was found in tumours with methylation as compared with those with unmethylated CACNA2D3. In-vitro exogenous CACNA2D3 expression inhibited cell growth and adhesion and upregulated p21 and p27 expression in gastric cancer cell lines with inverse effects with CACNA2D3 small interfering RNA treatment (Wanajo et al, 2008).

Given the evidence of the biological properties of the CACNA2D3 subunit and its possible role in human malignant disease, we investigated the expression and epigenetic regulation of CACNA2D3 in human breast cancer cell lines as well as in clinical samples of primary and metastatic breast cancer.

\section{MATERIALS AND METHODS}

\section{Breast cancer cell lines}

Breast carcinoma cell lines (MDA-MB-231, MDA-MB-436, MDAMB-453, MDA-MB-468, MCF-7, T47D, BT549, HCC1937, SKBR3, ZR75-1) were obtained from the Cancer Research UK Cell services (Claire Hall Laboratories, South Mimms, UK). All cells were maintained in Dulbecco's Modified Eagle Medium supplemented with $5 \mathrm{~mm} \mathrm{~L}$-glutamine and $10 \%$ fetal bovine serum.

For pharmacological demethylation, cells were treated with $5 \mu \mathrm{m}$ azacytidine (AZA; Sigma, Gillingham, UK) for 7 days. Cells were split every 2-3 days with the addition of fresh drug. After drug treatment, cells were harvested for qPCR.

\section{Tumours}

The study was performed with local research ethical approval. Primary breast carcinomas were from the pathology archives of $S$ Croce General Hospital, Cuneo, Italy and all central nervous system (CNS) metastatic carcinomas were from the neuropathology archives of Imperial College Healthcare NHS Trust, London, UK. All cases were histologically confirmed as breast carcinoma, and analysed for expression of OEstrogen receptor- $\alpha$ (ER), progesterone receptor (PgR) and HER2 according to local protocols. As normal tissue controls, we used genomic DNA isolated from five pooled normal breasts obtained at reduction mammoplasty.

\section{Nucleic acid isolation}

Genomic DNA and RNA were isolated from cell lines using commercially available kits (Qiagen, Venlo, The Netherlands). Genomic DNA was isolated from archival cases in Cuneo by proteinase $\mathrm{K}$ digestion of $10 \mu \mathrm{m}$ sticks cut from formalin-fixed paraffin-embedded tissue sections using standard xylene-phenol protocol. Total RNA was isolated from paraffin tissues using the RecoverAll Total Nucleic Acid Isolation kit (Ambion, Foster City, CA, USA).

\section{Pyrosequencing analysis}

Methylation in the $\mathrm{CpG}$ island of the CACNA2D3 genes was analysed using Pyrosequencing to quantify the degree of methylation at each $\mathrm{CpG}$ site by measurement of the ratio between $\mathrm{T}$ and $\mathrm{C}$. Primer sequences were as follows:

Forward primer $5^{\prime}$-GGTTAAGGATATTGGAGTTTT- $3^{\prime}$ Reverse primer 5'-biot-CCTCTAACAACAACAACC-3' Amplicon length $128 \mathrm{bp}$

PCR conditions were $95^{\circ} \mathrm{C}$ for $10 \mathrm{~min}, 95^{\circ} \mathrm{C}$ for $30 \mathrm{~s} / 52{ }^{\circ} \mathrm{C}$ for $30 \mathrm{~s} /$ $72{ }^{\circ} \mathrm{C}$ for $40 \mathrm{~s}$ for $40 \mathrm{cycles}, 72{ }^{\circ} \mathrm{C}$ for $7 \mathrm{~min}$. PCR products were then analysed by pyrosequencing using the Sample Prep kit (Diatech, Jesi, Italy) and the forward primer for sequencing. After pyrosequencing, analysis of percentage methylation at each $\mathrm{CpG}$ site was done using Pyromark QCpG Software (Qiagen). Placental DNA was used as negative control of methylation ( $0 \%$ average methylation) and a commercial methylated DNA (Millipore, Watford, UK) was used as positive control ( $98 \%$ average methylation).

\section{Analysis of gene expression}

For qPCR analysis, $25 \mu \mathrm{l}$ PCR reactions were performed using $50 \mathrm{ng}$ of cDNA obtained by reverse transcription of $1 \mu \mathrm{g}$ of RNA. Amplification and analysis were done according to the manufacturer's protocol in 96-well plates in an ABI PRISM 7000 Sequence Detection System (Applied Biosystems, Life Technologies Italia, Monza, Italy) and the pre-cast TaqMan Gene Expression Assays' (Applera; https://products.appliedbiosystems.com/) for CACNA2D3 (Hs00218157_m1). Quantification of target transcripts was performed in comparison to the reference transcript $\beta 2$ microglobulin (Hs99999907_m1), using the ' $\delta$ - $\delta$ Ct' method.

\section{Statistical analysis}

Methylation status of the $\mathrm{CpG}$ island of CACNA2D3 and presence of or sites of metastasis were assessed for association using Fisher's exact test.

\section{RESULTS}

\section{CACNA2D3 CpG island imethylation in breast cancer cell lines}

A CpG island is located in the $5^{\prime}$ regulatory sequences of CACNA2D3. We used pyrosequencing to analyse methylation in this $\mathrm{CpG}$ island in the cell line panel. There was dense methylation in MDA-MB-231 and MDA-MB-453 and low-level methylation in T47D, but no evidence of methylation in normal breast epithelial cells or in the remaining cell lines in the panel (Figure 1A). Using qPCR, we studied expression of CACNA2D3 mRNA in a panel of breast carcinoma cell lines. CACNA2D3 mRNA was abundantly expressed in the majority of cell lines analysed but was downregulated in MDA-MB-231, MDA-MB-453 and T47D (Figure 1B) confirming a good correlation between methylation and downregulated expression.

To confirm the role of methylation in silencing expression of CACNA2D3, we grew MDA-MB-436 (unmethylated) and MDAMB-453 (methylated) cells in the presence of the demethylating agent AZA and analysed CACNA2D3 mRNA using qPCR. In MDAMB-453, exposure to AZA caused a strong upregulation of CACNA2D3 mRNA but AZA had no effect in MDA-MB-436 (Figure 2A). Consistent with upregulation, pyrosequencing revealed that AZA caused demethylation in the CpG island of MDA-MB-453 but the low level of methylation in MDA-MB-436 was unaffected (Figure 2B).

\section{The CACNA2D3 CpG island is methylated in metastatic breast cancer}

Next, we wished to determine whether the CACNA2D3 CpG island is methylated in clinical cases of breast cancer. In particular, we wished to determine whether methylation is present in metastatic 
A

\begin{tabular}{|c|c|c|c|c|c|}
\hline & & ER & $\mathrm{PgR}$ & Her-2 & $\begin{array}{llllllllll}1 & 2 & 3 & 4 & 5 & 6 & 7 & 8 & 9 & 10\end{array}$ \\
\hline MDA-MB-231 & Basal & - & - & - & 0000000 \\
\hline MDA-MB-436 & Basal & - & - & - & 0000 \\
\hline MDA-MB-453 & Luminal & - & - & Amp & \\
\hline MDA-MB-468 & Basal & - & - & - & \\
\hline MCF7 & Luminal & + & + & - & $00 \mathrm{C}$ \\
\hline T47D & Luminal & + & + & - & 0000 \\
\hline BT549 & Basal & - & - & - & 0000 \\
\hline HCC1937 & Basal & - & - & - & 0000 \\
\hline SKBR3 & Luminal & - & - & Amp & 0000000 \\
\hline ZR75.1 & Luminal & + & - & $\mathrm{N}$ & 7 \\
\hline lormal & & & & & \\
\hline
\end{tabular}

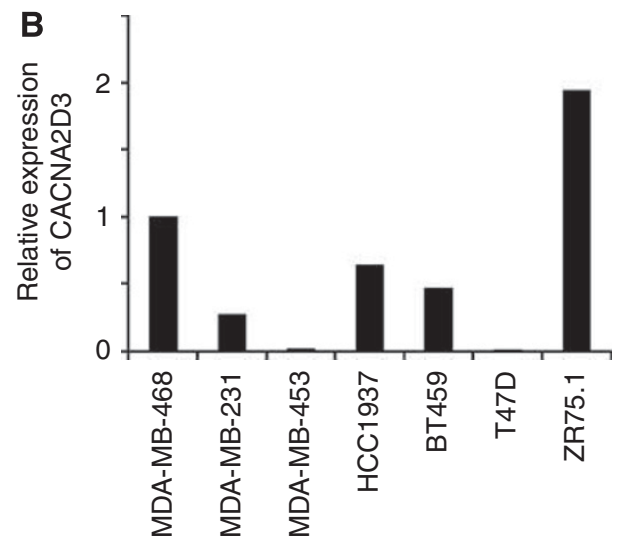

Figure I Methylation-dependent transcriptional silencing of CACNA2D3 in breast cancer cell lines. (A) Quantitative pyrosequencing analysis of the CACNA2D3 CpG island in breast carcinoma cell lines. Pyrosequencing was done as described in Materials and Methods. The level of methylation is represented by the intensity of shading in the circles, each of which represents an individual CpG dinucleotide in the amplified fragment. The mean CpG methylation in the amplified fragment, together with the MSP analysis is also shown. Abbreviations: $\mathrm{Amp}=$ Amplified; $\mathrm{ER}=$ Oestrogen receptor, $\mathrm{PgR}=$ Progesterone receptor. $(\mathbf{B}) \mathrm{qPCR}$ analysis of CACNA2D3 expression in breast cancer cell lines. qPCR was performed as described in Materials and Methods. Expression is shown relative to MDA-MB-468.
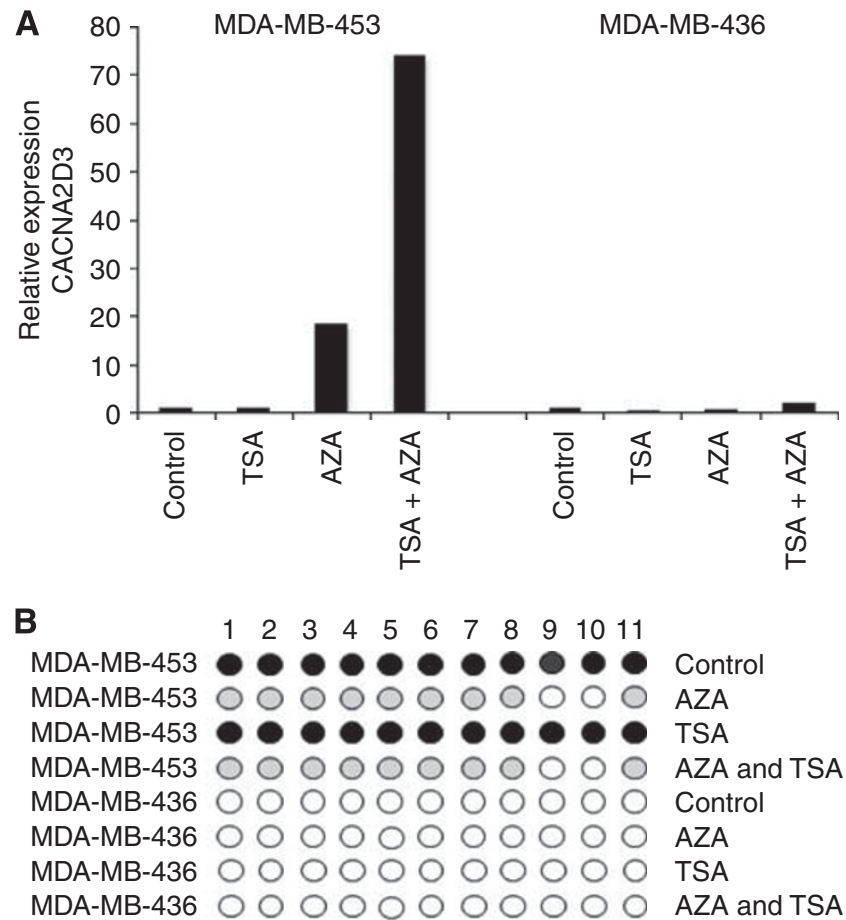

$$
\text { ○-5\% ○ } 6-10 \% \quad \bigcirc 11-20 \% \quad 21-50 \% \quad \text { ○ } 51-100 \%
$$

Figure 2 Demethylation reactivates expression of CACNA2D3. (A) MDA-MB-453 and MDA-MB-436 cells were grown in the presence of AZA, trichostatin A (TSA) or both. Expression of CACNA2D3 was determined by qPCR. (B) AZA-dependent demethylation of the CACNA2D3 CpG island correlates with re-expression of CACNA2D3. Cells were treated with AZA and TSA as above. CPG methylation was determined by pyrosequencing and the level of methylation is represented by the intensity of shading in the circles, each of which represents an individual CPG dinucleotide in the amplified fragment.

breast cancer. Accordingly, using pyrosequencing, we analysed in detail methylation in a series of 18 histological confirmed CNS breast cancer metastases (Figure 3). The fragment of the CACNA2D3 CpG island analysed by pyrosequencing was entirely unmethylated in normal breast epithelium, with no methylation detected at any of the 11 analysed CpG dinucleotides (Figure 3). In the CNS metastases, the level of methylation at each $\mathrm{CpG}$
A $\mathrm{CpG}$

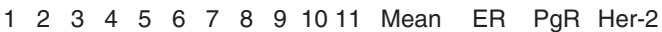
Brain met $\bigcirc 00000000001.1$ Brain met $000000000002.0+-$ Brain met 000000000007.4 - $\quad$ Brain met $000000000000++$ + Brain met 000000000002.3 - - + Brain met $000000000002.9+{ }_{-}+$ Brain met $\bigcirc 00000000005.2-++$ Brain met $\bigcirc 00000000000.6+++$ Brain met $\bigcirc \bigcirc \bigcirc$ Brain met 000000000006.3 - $\quad$ - Brain met $000000000005.2+-+$ Brain met $000000000003.8-++$ Brain met $\bigcirc 00000000004.4+t_{+}+$ Brain met $000000000002.0+$ + + Brain met $000000000001.6-{ }_{-}-$ Brain met $00000000025.1+$ Brain met Normal 000000000000

$0-5 \% \bigcirc 6-10 \% \bigcirc 11-20 \% \cdot 21-50 \% \cdot 51-100 \%$

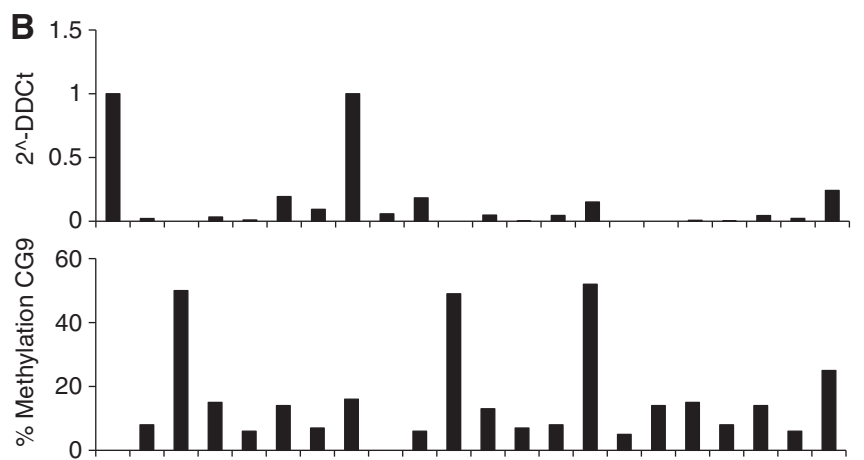

Figure 3 (A) The CACNA2D3 CpG island is methylated in metastatic breast cancer. The figure shows pyrosequencing analysis of 17 CNS metastases derived from CNS metastases derived from primary breast carcinomas. CpG methylation was determined by pyrosequencing and the level of methylation is represented by the intensity of shading in the circles, each of which represents an individual CpG dinucleotide in the amplified fragment. The ER, PgR and Her2 status is also shown for each case. (B) Methylation-associated downregulation of CACNA2D3 in breast cancers is associated with $\mathrm{CpG}$ island methylation. The upper panel shows qPCR analysis of CACNA2D3 in a series of primary breast carcinomas (each of which later relapsed with loco-regional or distant metastatic disease). The lower panel shows percentage methylation at CpG9 in the fragment of the CpG island analysed by pyrosequencing. 
Table I Clinico-pathological characteristics of all I 42 patients

\begin{tabular}{lr}
\hline \multicolumn{2}{c}{ Age (at primary diagnosis) median: 64 (range: 36-87) } \\
\hline Tumour size: $n$ (\%) & $21(15)$ \\
$<20 \mathrm{~mm}$ & $18(13)$ \\
$20-49 \mathrm{~mm}$ & $0(0)$ \\
$50 \mathrm{~mm}$ & $103(72)$ \\
NA & \\
Tumour grade: $n$ (\%) & $8(6)$ \\
Grade I & $102(72)$ \\
Grade II & $22(15)$ \\
Grade III & $10(7)$ \\
NA & \\
Nodal status: $n$ (\%) & $53(37)$ \\
Positive & $68(48)$ \\
Negative & $21(15)$ \\
NA & \\
Hormone receptor status: $n$ (\%) & $85(60)$ \\
ER + ve and PR + ve & $41(29)$ \\
ER + ve and PR -ve/unknown & $13(9)$ \\
ER -ve and PR -ve/unknown & $3(2)$ \\
ER unknown and PR unknown missing & \\
HER2 & $20(0.14)$ \\
Positive & $113(0.80)$ \\
Negative & $9(0.06)$ \\
NA &
\end{tabular}

Abbreviations: $E R=$ oestrogen receptor; $N A=$ not available; $P R=$ progesterone receptor.

dinucleotide varied markedly within individual lesions (Figure 3). The most frequently and densely methylated CpGs in the amplified fragment were dinucleotides 9 and 10 (Figure 3). With a cutoff of $7 \%$ methylation, 8 out of $18(44 \%)$ CNS metastases were positive for methylation at CpG9 and 8 out of 18 (44\%) positive for methylation at CpG10. These results show that the CACNA2D3 $\mathrm{CpG}$ island is methylated in metastatic breast cancer lesions. To confirm that expression of CACNA2D3 was affected by CpG island methylation, we used qPCR to measure steady-state mRNA levels in 21 primary breast carcinomas that later relapsed with either loco-regional or distant metastatic disease. CACNA2D3 mRNA was downregulated in the majority of these cases (Figure 3B). Using pyrosequencing we analysed CACNA2D3 CpG island methylation. There was increased methylation in almost all cases with downregulation of the mRNA (Figure 3B).

\section{CACNA2D3 CpG island methylation predicts site-specific relapse in primary breast carcinomas treated with endocrine therapy}

The presence of CACNA2D3 CpG island methylation in CNS metastatic breast carcinomas prompted us to determine whether methylation in primary carcinomas is predictive of future recurrence and/or metastasis. The clinico-pathological parameters of the study population are shown in Table 1. Again using pyrosequencing, we analysed an archival series of 142 cases from our clinical practice, which contained both ER-positive, ERnegative and triple negative cancers. Patients were treated adjuvantly according to normal protocols of clinical care. As seen with the CNS lesions, the level of methylation was variable between specific CpG dinucleotides in individual patients (see Figure 4 for representative pyrosequencing data). Using a cutoff of mean $7 \%$ methylation for the amplified fragment analysed by pyrosequencing, 38 out of $142(27 \%)$ cases were positive for CACNA2D3 methylation. We wished to explore in more detail, whether the presence of methylation in the CACNA2D3 CpG island in primary

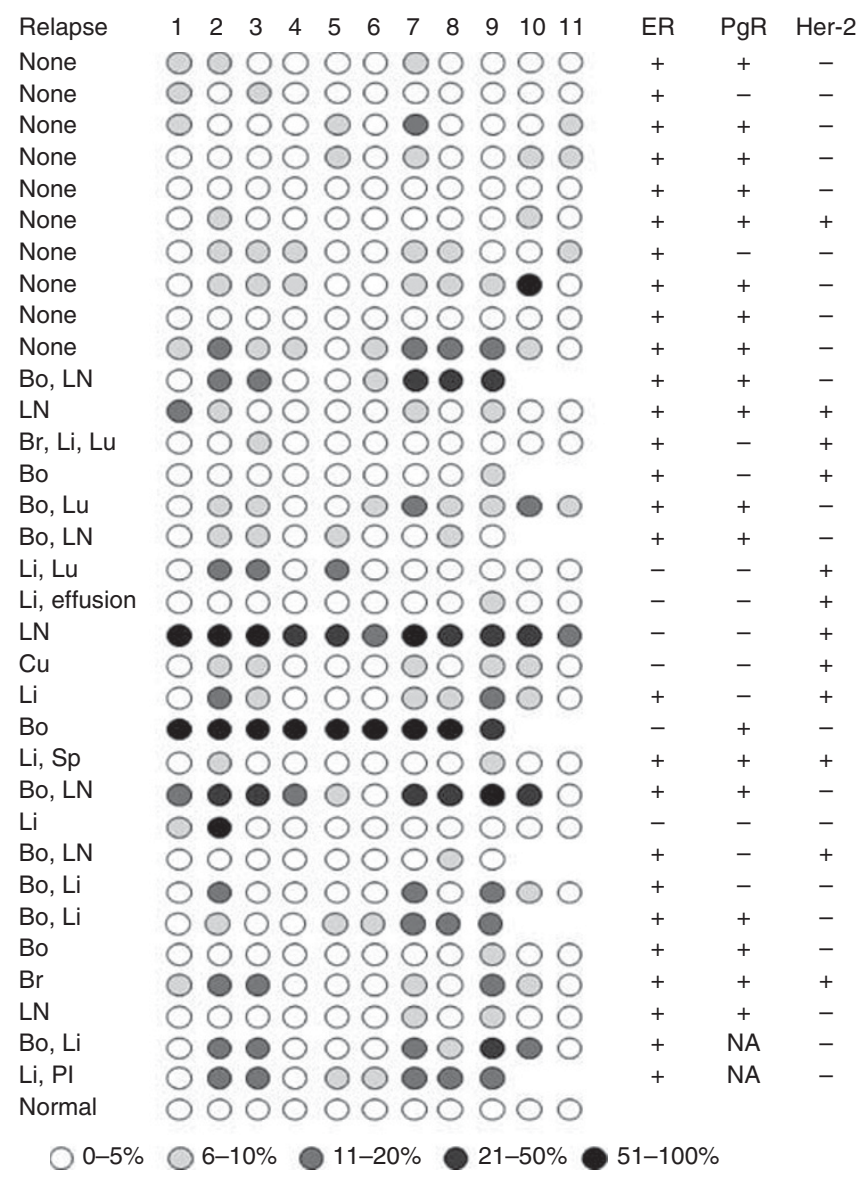

Figure 4 The CACNA2D3 CpG island is methylated in primary breast cancer. The figure shows representative pyrosequencing analysis of individual primary breast carcinomas which were either non-relapsing or relapsed at various distant organ sites. CpG methylation was determined by pyrosequencing and the level of methylation is represented by the intensity of shading in the circles, each of which represents an individual CPG dinucleotide in the amplified fragment. The ER, PgR and Her2 status is also shown for each case. Abbreviations: $\mathrm{Bo}=$ bone; $\mathrm{Br}=$ brain; $\mathrm{Cu}=$ cutaneous; $\mathrm{Li}=$ liver; $\mathrm{LN}=$ lymph node; $\mathrm{Lu}=$ lung; $\mathrm{PI}=$ pleura; $\mathrm{Sp}=$ spleen; $\mathrm{NA}=$ not available.

cancers was associated with an increased probability of future metastasis. We therefore excluded those cases in which full clinicopathological parameters were not available and those patients who had been lost to follow-up and determined the frequency of metastatic relapse as a function of CACNA2D3 CpG island methylation. We then performed further analysis in 100 of the cases for which we had complete clinic-pathological information, treatment and clinical outcome. These 100 cases comprised of ERpositive, tamoxifen-treated patients, clinic-pathological details of this group are presented in Table 2. Detailed analysis of each individual $\mathrm{CpG}$ dinucleotide within the amplified fragment of the CACNA2D3 CpG island revealed that methylation at CpG9 was a sensitive predictive biomarker of future metastatic relapse and a specific discriminator between cases which did and did not relapse (Figures 4 and 5A). At the time of analysis, 51 patients had relapsed with either loco-regional or distant metastatic disease. Applying a methylation cutoff of $7 \%$ at CpG9, the frequency of methylation was significantly higher in cases that relapsed than in cases with no relapse: 21 out of 61 (34\%) methylated in nonrelapsing cases $v s 30$ out of $39(77 \%)$ methylated in relapsing cases; $P<0.0001$. Next, we asked in the same patient population whether methylation in the CACNA2D3 $\mathrm{CpG}$ island in primary breast carcinomas affected the risk of later metastasis in specific 
anatomical sites. Metastasis to liver and lung was significantly more common in primary carcinomas with methylated CACNA2D3 than in cases lacking methylation, $P=0.012$ and $P=0.02$, respectively (Figure $5 \mathrm{~B}$ ). Metastasis to bone and brain was also more common in primary cancers, with methylation in CACNA2D3 but because of the small number of cases these did not reach statistical significance (Figure 5B). There was no evidence that metastasis to skin or lymph nodes was increased in cases with CACNA2D3 CpG island methylation (Figure 5B).

\section{DISCUSSION}

Calcium ion channels mediate many biological processes potentially relevant to the malignant process including metastasis (Patton et al, 2003; Huang et al, 2004). CACNA2D3 in particular has a number of properties consistent with a tumour and/or

Table 2 Clinico-pathological characteristics of I00 ER-positive tamoxifen-treated patients

\begin{tabular}{|c|c|}
\hline \multicolumn{2}{|c|}{ Age (at primary diagnosis) median: 64.5 (range: 36-87) } \\
\hline \multicolumn{2}{|l|}{ Tumour size: $\mathrm{n}(\%)$} \\
\hline$<20 \mathrm{~mm}$ & $16(16)$ \\
\hline $20-49 \mathrm{~mm}$ & $17(17)$ \\
\hline$>50 \mathrm{~mm}$ & $0(0)$ \\
\hline NA & $67(67)$ \\
\hline \multicolumn{2}{|l|}{ Tumour grade: $\mathrm{n}(\%)$} \\
\hline Grade I & $6(6)$ \\
\hline Grade II & $75(75)$ \\
\hline Grade III & $13(13)$ \\
\hline NA & $6(4)$ \\
\hline \multicolumn{2}{|l|}{ Nodal status: n (\%) } \\
\hline Positive & $38(38)$ \\
\hline Negative & $46(46)$ \\
\hline NA & $16(16)$ \\
\hline \multicolumn{2}{|c|}{ Hormone receptor status: $\mathrm{n}(\%)$} \\
\hline$E R+v e$ and $P R+v e$ & $68(68)$ \\
\hline$E R+v e$ and $P R-v e$ & $32(32)$ \\
\hline \multicolumn{2}{|l|}{ HER2 } \\
\hline Positive & ||$(||)$ \\
\hline Negative & $84(84)$ \\
\hline NA & $5(5)$ \\
\hline
\end{tabular}

Abbreviations: $E R=$ oestrogen receptor; $N A=$ not available; $P R=$ progesterone receptor. metastasis suppressor function as demonstrated by its ectopic expression inhibiting cell growth and adhesion in gastric cancer cell lines, whereas knockdown with inhibitory RNA resulted in increased proliferation (Wanajo et al, 2008). Consistent with these in-vitro findings, methylation was more common in gastric cancer tissue as compared with normal gastric tissue and in those cancers where methylation was present it was associated with a shorter overall survival (Wanajo et al, 2008). Further evidence supporting a role of CACNA2D3 lung cancer, renal cell cancer, neuroblastoma and osteosarcoma has been previously reported (Hanke et al, 2001; De Preter et al, 2006; Tai et al, 2006; Thorell et al, 2009; Xiong et al, 2009). With regard to breast cancer, CACNA2D3 lies in 3p21 a region implicated in sporadic breast cancer development (Buchhagen et al, 1994), but no study has addressed these issues in breast cancer. We show in the present work that CACNA2D3 is subject to epigenetic regulation in breast cancer cell lines and primary and metastatic lesions via aberrant methylation in the CpG island located in the regulatory elements of the gene, consistent with such a tumour suppressor function in breast cancer and consistent with the previous data in other tumour types (Hanke et al, 2001; De Preter et al, 2006; Tai et al, 2006).

We first demonstrated using quantitative analysis (pyrosequencing) that CACNA2D3 expression varies with the methylation status of the CPG island. There was dense methylation in MDAMB-231 and MDA-MB-453 cell lines with concomitant downregulated expression. In contrast, the $\mathrm{CpG}$ island was unmethylated in normal breast epithelium and the remaining cell lines examined. Interestingly, both cell lines with methylation-dependent silencing of CACNA2D3 were ER-negative, although several other ER-negative cell lines did not show methylation and expressed CACNA2D3. In the clinical series analysed, the CACNA2D3 CpG island was methylated in both ER-positive and ER-negative cases. Expression of CACNA2D3 mRNA was efficiently restored by demethylation with AZA. Together, these observations are consistent with methylation-dependent transcriptional silencing being the mechanistic basis of CACNA2D3 downregulation in breast cancer.

We then analysed CACNA2D3 methylation in three wellcharacterised clinical series of breast cancers. The first comprised a panel of CNS metastases derived from patients with breast cancer and all confirmed by histopathology to be metastatic deposits of breast cancer. Detailed, quantitative pyrosequencing analysis of genomic DNA from this series revealed that methylation in the CpG island of CACNA2D3 was heterogeneous within individual cancers, as evidenced by variable levels of methylation at specific CpG dinucleotides within the analysed fragment. However, methylation at CpG9 within the amplified fragment was most
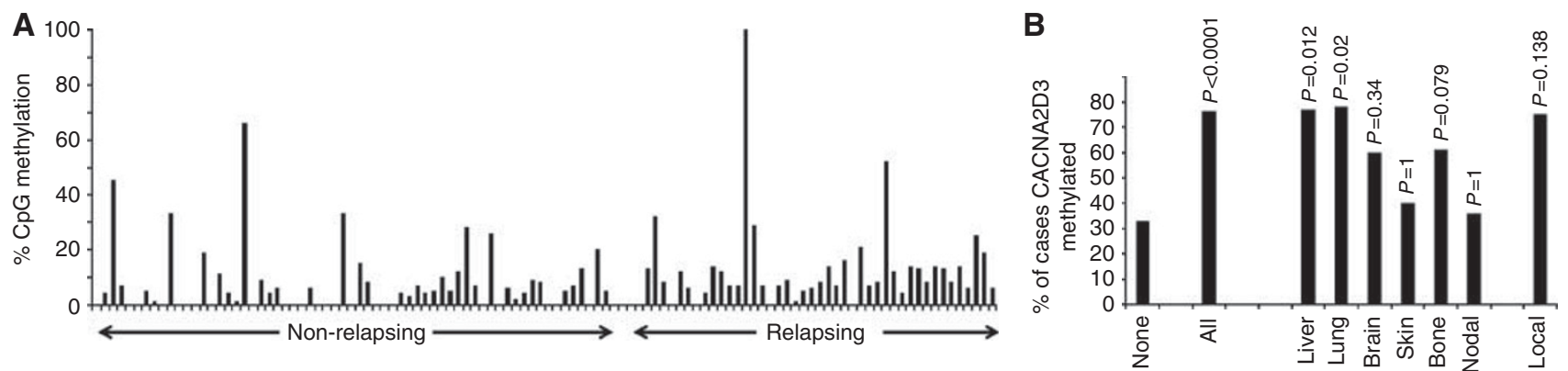

Figure 5 Methylation in the CACNA2D3 CpG island in primary breast cancers treated adjuvantly with endocrine therapy is associated with increased risk of visceral metastasis. (A) Percentage methylation at CPG9 in non-relapsing and relapsing primary breast carcinomas. The figure shows percentage methylation, determined by pyrosequencing, in individual cases which remained disease free or which later relapsed at various distant organ sites. (B) Methylation in CACNA2D3 predicts visceral relapse in tamoxifen-treated primary breast carcinomas. The figure shows the percentage of cases with methylation in primary cancers that did not relapse ('none'), in all relapsing cases ('all') and in the indicated metastatic sites. The P-value, calculated as described in Materials and Methods is shown above each column. 
strongly associated with brain metastatic lesions. The presence of a relatively high frequency of methylation in CACNA2D3 in CNS metastases prompted us to determine whether methylation in primary breast carcinomas is associated with increased risk of recurrence and/or metastasis. In a series of 100 predominantly ER-positive primary breast carcinomas treated adjuvantly with tamoxifen, we demonstrated that in primary cancers with CACNA2D3 CpG methylation, there is a significantly increased risk of recurrence, particularly at visceral sites of liver and lung, whereas there was no increased risk of nodal metastases. Our results are consistent with studies in gastric cancer, which have suggested that downregulation of CACNA2D3 is associated with clinically more aggressive disease.

Previous in-vitro data in non-small cell lung cancer has shown that the A2D2 subunit can induce apoptosis by disrupting mitochondrial membrane integrity via its effect on intracellular calcium (Carboni et al, 2003). However, the loss of the A2D2 subunit in vitro is associated with abnormal growth, abnormalities in cell adhesion and downregulation of key cell-cycle regulators (Wanajo et al, 2008). The abnormalities in cell adhesion may be linked to the fact that the A2D3 subunit contains a von Willebrand A domain (Whittaker and Hynes, 2002), which is known to contain metal-ion-dependent adhesion sites responsible for binding to extracellular matrix protein. Therefore, it appears that the two $\alpha_{2} \delta$ subunits implicated in malignant disease have their own unique function and are important for normal functioning of calcium channels. Further work is required to understand the normal physiological role of CACNA2D3 in a non-excitable cells such as breast epithelium, as well as an understanding of the pathomolecular effects of perturbation or loss of these calcium channel subunits.

Interestingly, chromatin immunoprecipitation-based assay in MCF-7 cells has shown that, following treatment with estradiol, CACNA2D3 is negatively regulated by the co-activator steroid receptor co-activator-3 via ER (Labhart et al, 2005). Steroid receptor co-activator-3 is known to be an oncogene, which is involved in mammary tumourigenesis, endocrine resistance and is associated with a poorer outcome in breast cancer (Gojis et al, 2010). Furthermore, it can modulate cell motility and invasion

\section{REFERENCES}

Bai J, Uehara Y, Montell DJ (2000) Regulation of invasive cell behavior by taiman, a Drosophila protein related to AIB1, a steroid receptor coactivator amplified in breast cancer. Cell 103: 1047-1058

Becchetti A (2011) Ion channels and transporters in cancer. 1. Ion channels and cell proliferation in cancer. Am J Physiol Cell Physiol 301: C255-C265

Berridge MJ, Bootman MD, Roderick HL (2003) Calcium signalling: dynamics, homeostasis and remodelling. Nature Rev Mol Cell Biol 4: 517-529

Buchhagen DL, Qiu L, Etkind P (1994) Homozygous deletion, rearrangement and hypermethylation implicate chromosome region 3p14.3-3p21.3 in sporadic breast-cancer development. Int J Cancer 15: 473-479

Carboni GL, Gao B, Nishizaki M, Xu K, Minna JD, Roth JA, Ji L (2003) CACNA2D2-mediated apoptosis in NSCLC cells is associated with alterations of the intracellular calcium signaling and disruption of mitochondria membrane integrity. Oncogene 22: 615-626

Davies A, Hendrich J, Van Minh AT, Wratten J, Douglas L, Dolphin AC (2007) Functional biology of the alpha(2)delta subunits of voltage-gated calcium channels. Trends Pharmacol Sci 28: 220-228

De Preter K, Vandesompele J, Heimann P, Yigit N, Beckman S, Schramm A, Eggert A, Stallings RL, Benoit Y, Renard M, De Paepe A, Laureys G, Påhlman S, Speleman F (2006) Human fetal neuroblast and neuroblastoma transcriptome analysisconfirms neuroblast origin and highlights neuroblastoma candidate genes. Genome Biol 7: R84. 1-17

Gojis O, Rudraraju B, Gudi M, Hogben K, Sousha S, RC Coombes, Cleator S, Palmieri C (2010) The role of SRC-3 in human breast cancer. Nat Rev Clin Oncol 7: 83-89

Hanahan D, Weinberg RA (2000) The hallmarks of cancer. Cell 100: 57-70 in breast cancer cell lines (Bai et al, 2000; Li et al, 2008a, b). This phenotype could be via its ability to downregulate the expression of CACNA2D3.

In summary, the association of methylation in the CACNA2D3 $\mathrm{CpG}$ island with breast cancer metastasis and in particular visceral disease implies that analysis of this gene may be utilised as a biomarker for metastasis and warrants evaluation in larger independent clinical series. Further work is required to understand the normal physiological role of CACNA2D3 in a non-exciable cells such as breast epithelium, and the molecular effects and mechanisms underlying CACNA2D2 role in malignant disease mediated regulation of cell proliferation and cell death in the pathogenesis of lung cancers and other human cancers.

\section{ACKNOWLEDGEMENTS}

Carlo Palmieri is the recipient of a Cancer Research UK Clinician Scientist award and also acknowledges support from Imperial College Healthcare Charity. Ondrej Gojis is in part funded by a grant from the Ministry of Education of the Czech Republic (Project 'Oncology' MSM 0021620808) and is also a recipient of the Translational Research Fellowship from the European Society of Medical Oncology and a fellowship from the European Society for Surgical Oncology. The Division of Cancer at Imperial College London, Imperial College Healthcare NHS Trust is an Experimental Cancer Medicine Centre that is supported by funds from Cancer Research UK and the Department of Health (C37/A7283) and also forms part of the Imperial Cancer Research UK Centre (C42671/A12196). We thank Professor Gerry Thomas, Sarah Chilcott-Burns and the Imperial College Healthcare NHS Trust, Human Biomaterials Resource Centre (Tissue Bank). Nelofer Syed acknowledges support from the Brain Tumour Research Campaign (BTRC). We thank De Federico Roncaroli for his contribution to the work.

\section{Conflict of interest}

The authors declare no conflict of interest.

Hanke S, Bugert P, Chudek J, Kovacs G (2001) Cloning a calcium channel alpha2delta-3 subunit gene from a putative tumor suppressor gene region at chromosome 3 p21.1 in conventional renal cell carcinoma. Gene 264: $69-75$

Huang JB, Kindzelskii AL, Clark AJ, Petty HR (2004) Identification of channels promoting calcium spikes and waves in HT1080 tumor cells: their apparent roles in cell motility and invasion. Cancer Res 64: 2482-2489

Kim SG, Chan AO, Wu TT, Issa JP, Hamilton SR, Rashid A (2003) Epigenetic and genetic alterations in duodenal carcinomas are distinct from biliary and ampullary carcinomas. Gastroenterology 124: 1300-1310

Labhart P, Karmakar S, Salicru EM, Egan BS, Alexiadis V, O’Malley BW, Smith CL (2005) Identification of target genes in breast cancer cells directly regulated by the SRC-3/AIB1 coactivator. Proc Nat Assoc Sci 102: 1339-1344

Li C, Liang YY, Feng XH, Tsai SY, Tsai MJ, O’Malley BW (2008a) Essential phosphatases and a phospho-degron are critical for regulation of SRC-3/AIB1 coactivator function and turnover. Mol Cell 31: 835-849

Li LB, Louie MC, Chen HW, Zou JX (2008b) Proto-oncogene ACTR/AIB1 promotes cancer cell invasion by up-regulating specific matrix metalloproteinase expression. Cancer Lett 261: 64-73

Monteith GR, McAndrew D, Faddy HM, Roberts-Thomson SJ (2007) Calcium and cancer: targeting $\mathrm{Ca} 2+$ transport. Nat Rev Cancer 7: 519-530

Patton AM, Kassis J, Doong H, Kohn EC (2003) Calcium as a molecular target in angiogenesis. Curr Pharm Des 9: 543-551 
Paz MF, Wei S, Cigudosa JC, Rodriguez-Perales S, Peinado MA, Huang TH, Esteller M (2003) Genetic unmasking of epigenetically silenced tumor suppressor genes in colon cancer cells deficient in DNA methyltransferases. Hum Mol Genet 12: 2209-2219

Qin N, Yagel S, Momplaisir ML, Codd EE, D'Andrea MR (2002) Molecular cloning and characterization of the human voltage-gated calcium channel alpha(2)delta-4 subunit. Mol Pharmacol 62: 485-496

Qin YR, Fu L, Sham PC, Kwong DL, Zhu CL, Chu KK, Li Y, Guan XY (2008) Single-nucleotide polymorphism-mass array reveals commonly deleted regions at 3 p22 and 3 p14.2 associate with poor clinical outcome in esophageal squamous cell carcinoma. Int J Cancer 123: 826-830

Rizzuto R, Pinton P, Ferrari D, Chami M, Szabadkai G, Magalhães PJ, Di Virgilio F, Pozzan T (2003) Calcium and apoptosis: facts and hypotheses. Oncogene 22: 8619-8627

Rizzuto R, Pozzan T (2006) Microdomains of intracellular Ca2 + : molecular determinants and functional consequences. Physiol Rev 86: 369-408

Tai AL, Mak W, Ng PK, Chua DT, Ng MY, Fu L, Chu KK, Fang Y, Qiang Song Y, Chen M, Zhang M, Sham PC, Guan XY (2006) High-throughput loss-of-heterozygosity study of chromosome $3 \mathrm{p}$ in lung cancer using single nucleotide polymorphism markers. Cancer Res 66: 4133-4138

Thorell K, Bergman A, Carén H, Nilsson S, Kogner P, Martinsson T, Abel F (2009) Verification of genes differentially expressed in neuroblastoma tumours: a study of potential tumour suppressor genes. BMC Med Genomics 53: 1-13
Toyota M, Ho C, Ohe-Toyota M, Baylin SB, Issa JP (1999) Inactivation of CACNA1G, a T-type calcium channel gene, by aberrant methylation of its 5' CpG island in human tumors. Cancer Res 59: 4535-4541

Wanajo A, Sasaki A, Nagasaki H, Shimada S, Otsubo T, Owaki S, Shimizu Y, Eishi Y, Kojima K, Nakajima Y, Kawano T, Yuasa Y, Akiyama Y (2008) Methylation of the calcium channel-related gene, CACNA2D3, is frequent and a poor prognostic factor in gastric cancer. Gastroenterology 135: 580-590

Wei MH, Latif F, Bader S, Kashuba V, Chen JY, Duh FM, Sekido Y, Lee CC, Geil L, Kuzmin I, Zabarovsky E, Klein G, Zbar B, Minna JD, Lerman MI (1996) Construction of a 600-kilobase cosmid clone contig and generation of a transcriptional map surrounding the lung cancer tumor suppressor gene (TSG) locus on human chromosome 3p21.3: progress toward the isolation of a lung cancer TSG. Cancer Res 56: $1487-1492$

Whittaker CA, Hynes RO (2002) Distribution and evolution of von Willebrand/integrin A domains: widely dispersed domains with roles in cell adhesion and elsewhere. Mol Biol Cell 13: 3369-3387

Xiong W, Niu PY, Zhu WT, Chen J (2009) Tenascin-C as a prognostic biomarker in osteosarcoma? Chin Med J 122: 2737-2743

Yuasa Y, Nagasaki H, Akiyama Y, Hashimoto Y, Takizawa T, Kojima K, Kawano T, Sugihara K, Imai K, Nakachi K (2009) DNA methylation status is inversely correlated with green tea intake and physical activity in gastric cancer patients. Int J Cancer 124: 2677-2682

This work is published under the standard license to publish agreement. After 12 months the work will become freely available and the license terms will switch to a Creative Commons Attribution-NonCommercial-Share Alike 3.0 Unported License. 